\title{
科尔沁沙地优势固沙灌木叶片氮磷化学计量内稳性
}

\author{
宁志英 ${ }^{1,2}$ 李玉霖 $^{1^{*}}$ 杨红玲 ${ }^{1,2}$ 张子谦 ${ }^{1,2}$
}

${ }^{1}$ 中国科学院西北生态环境资源研究院奈曼沙漠化研究站, 兰州 730000 $^{2}$ 中国科学院大学, 北京 100049

\begin{abstract}
摘 要 为科学认识科尔沁沙地优势固沙灌木的生态适应性和固沙植被演变规律, 该研究对科尔沁沙地流动沙丘、半固定沙 丘、固定沙丘和丘间低地的优势固沙灌木小叶锦鸡儿(Caragana microphylla)和盐蒿(Artemisia halodendron)进行野外调查, 研 究了这两种固沙灌木的叶片氮 $(\mathrm{N})$ 、磷 $(\mathrm{P})$ 化学计量特征、灌从土壤养分状况以及内稳性特征。结果表明: 1)与盐蒿相比, 灌木 小叶锦鸡儿具有较高的叶片 $\mathrm{N}$ 含量及 $\mathrm{N}: \mathrm{P}$, 而 $\mathrm{P}$ 含量仅为盐蒿的 $1 / 2 ; 2)$ 两种优势固沙灌木灌丛下土壤的全 $\mathrm{N}$ 、全 $\mathrm{P}$ 含量及速效 $\mathrm{N}$ 、 速效 $\mathrm{P}$ 含量高于该地区土壤的平均水平, 小叶锦鸡儿灌丛下土壤养分含量显著高于盐蒿灌从下土壤; 3)盐蒿叶片 $\mathrm{N} 、 \mathrm{P}$ 化学计量 内稳性指数 $(H)$ 表现为 $H_{\mathrm{P}}>H_{\mathrm{N}: \mathrm{P}}>H_{\mathrm{N}}$, 说明盐蒿更易受土壤 $\mathrm{N}$ 的限制; 小叶锦鸡儿叶片 $\mathrm{N} 、 \mathrm{P}$ 化学计量内稳性指数表现为 $H_{\mathrm{N}: \mathrm{P}}>$ $H_{\mathrm{N}}>H_{\mathrm{P}}$, 意味着小叶锦鸡儿更易受土壤 $\mathrm{P}$ 的限制。在 $\mathrm{N}$ 含量较低的沙化草地, $H_{\mathrm{N}}$ 较高的固沙灌木小叶锦鸡儿比盐蒿更具生长 优势，对于该地区生态恢复及保护具有不可替代的作用。然而，小叶锦鸡儿额外吸收的 $\mathrm{N}$, 使其生长过程可能易受 $\mathrm{P}$ 的限制, 因此在沙地恢复过程中应注意土壤P的供应。
\end{abstract}

关键词 科尔沁沙地; 灌木; 叶片; 土壤; 化学计量内稳性

宁志英, 李玉霖, 杨红玲, 张子谦 (2019). 科尔沈沙地优势固沙灌木叶片氮磷化学计量内稳性. 植物生态学报, 43, 46-54. DOI: 10.17521/cjpe.2018.0100

\section{Nitrogen and phosphorus stoichiometric homoeostasis in leaves of dominant sand-fixing shrubs in Horqin Sandy Land, China}

\author{
NING Zhi-Ying ${ }^{1,2}$, LI Yu-Lin ${ }^{1 *}$, YANG Hong-Ling ${ }^{1,2}$, and ZHANG Zi-Qian ${ }^{1,2}$ \\ ${ }^{1}$ Naiman Desertification Research Station, Northwest Institute of Eco-Environment and Resource, Chinese Academy of Sciences, Lanzhou 730000, China; and \\ ${ }^{2}$ University of Chinese Academy of Sciences, Beijing 100049, China
}

\begin{abstract}
Aims Sand-fixing shrubs play an irreplaceable role in ecological restoration and eco-environmental protection in arid and semiarid regions of northern China. Determination of the stoichiometric homoeostasis of dominant sand-fixing shrubs along soil nutrient gradients could provide insights into ecological adaptability and pattern of changes of sand-fixing vegetation in Horqin Sandy Land.

Methods We measured N and P concentrations in leaves of two dominant sand-fixing shrubs Caragana microphylla and Artemisia halodendron, and the total and available $\mathrm{N}$ and $\mathrm{P}$ concentrations in soils beneath the canopy of each shrub. The differences between the two shrubs in $\mathrm{N}$ and $\mathrm{P}$ concentrations and $\mathrm{N}: \mathrm{P}$ of leaves and soils as well as in stoichiometric homoeostasis were examined.

Important findings Caragana microphylla had higher leaf $\mathrm{N}$ concentration and lower leaf $\mathrm{P}$ concentration, thereby higher leaf N:P, than A. halodendron. Soils beneath the shrub canopies, regardless of the species, had higher total and available $\mathrm{N}$ and $\mathrm{P}$ concentrations relative to soils outside the canopy cover. Moreover, the total and available $\mathrm{N}$ and $\mathrm{P}$ concentrations in soils beneath the $C$. microphylla canopy were higher than that beneath the A. halodendron canopy. The stoichiometric homoeostasis indexes $(H)$ were ranked in the order of $H_{\mathrm{P}}>H_{\mathrm{N}: \mathrm{P}}>H_{\mathrm{N}}$ in A. halodendron and $H_{\mathrm{N}: \mathrm{P}}>H_{\mathrm{N}}>H_{\mathrm{P}}$ in C. microphylla, respectively, suggesting $\mathrm{N}$ limitation in $A$. halodendron and P limitation in C. microphylla. Therefore, Caragana microphylla could be used as nursing plants in degraded N-limiting soil because of high $H_{\mathrm{N}}$. However, due to excessive uptake of N, Caragana microphylla might suffer from $\mathrm{P}$ limitation, and adequate $\mathrm{P}$ supply should be considered during the restoration process in sandy land.
\end{abstract}

Key words Horqin Sandy Land; shrub; leaf; soil; stoichiometric homoeostasis

收稿日期Received: 2018-05-04 接受日期Accepted: 2018-12-24

基金项目: 国家自然科学基金(31270501和41471083)和国家重点研发计划(2016YFC0500907)。Supported by the National Natural Science Foundation of China (31270501 and 41471083), and the National Key R\&D Program of China (2016YFC0500907).

* 通信作者Corresponding author (liyl@lzb.ac.cn) 
Ning ZY, Li YL, Yang HL, Zhang ZQ (2019). Nitrogen and phosphorus stoichiometric homoeostasis in leaves of dominant sand-fixing shrubs in Horqin Sandy Land, China. Chinese Journal of Plant Ecology, 43, 46-54. DOI: 10.17521/cjpe.2018.0100

内稳性是指生物体在变化的环境中保持其自身 条件相对恒定的能力(Koojiman, 1995)。在化学计量 生态学范畴内, 环境中元素组成发生变化的情况下, 生物体保持体内元素组成相对稳定的能力叫做化学 计量内稳性(Reiners, 1986; Sterner \& Elser, 2002)。内 稳性反映了生物对环境变化的生理和生化的适应 (Hessen et al., 2004; Elser et al., 2010), 因此, 研究 生物的化学计量内稳性对我们解释物种的生态策略 和适应性有重要意义(Frost \& Elser, 2002; Jeyasingh et al., 2009)。

碳 $(\mathrm{C})$ 、氮 $(\mathrm{N})$ 、磷 $(\mathrm{P})$ 是陆地生态系统最重要的 生源元素, 对生态系统结构和功能具有重要作用 (Westheimer, 1987; Daufresne \& Loreau, 2001; Sundareshwar et al., 2003; Andersen et al., 2004)。C是植 物各生理生化过程的底物及能量来源, 而 $\mathrm{N} 、 \mathrm{P}$ 是植 物生长的主要限制养分(Elser et al., 2007)。植物根、 茎和叶等器官的 N、P含量受到土壤养分供应能力的 影响, 器官的 $\mathrm{N}$ 和 $\mathrm{P}$ 含量反映了土壤 $\mathrm{N}$ 和 $\mathrm{P}$ 的可利用 性(Vitousek, 1999; Aerts \& Chapin, 2000; Hobbie \& Vitousek, 2000), 例如Han等(2005)的研究指出, 土 壤与叶片 $\mathrm{P}$ 含量间存在正相关关系, 即较高的土壤 $\mathrm{P}$ 含量对应着较高的叶片 $\mathrm{P}$ 含量。但是, 由于植物具有 积极调控细胞成分或体内环境以保持元素组成相对 稳定的能力, 土壤养分改变对植物器官 $\mathrm{N} 、 \mathrm{P}$ 元素含 量的影响也取决于植物化学计量内稳性的强弱 (Persson \& Kato, 2010)。有研究发现, 植物器官N、 $\mathrm{P}$ 化学计量内稳性与群落稳定性、物种入侵等相关 (Yu et al., 2010; 蒋利玲等, 2017), 遗憾的是目前相 关研究严重不足, 而且对于土壤养分改变情况下植 物内稳性的研究仅限于很少的一些植物物种。目前, 国内外关于植物化学计量内稳性的研究多集中在草 本植物上(㡺强, 2009; Li et al., 2016), 对灌木化学 计量内稳性的研究仍较少。Chen等(2016)研究了我 国红壤山区芒萁(Dicranopteris dichotoma)的入侵机 制, 发现该物种化学计量内稳性较低是其成功入侵 贫㾑土壤的主要原因。Yan等(2015)研究了亚热带森 林生态系统中马尾松(Pinus massoniana) 逐渐消失的 原因, 发现马尾松 $\mathrm{P}$ 的内稳性低, 表明 $\mathrm{P}$ 限制作用导 致其演替后期无法存活。固沙灌木在北方干旱半干 旱区生态恢复和生态环境保护中具有不可替代的作
用(篗王龙等, 2015), 研究其化学计量内稳性特征对 于认识固沙灌木的生态适应性及固沙植被演替规律 具有重要意义。

在灌木的生长发育过程中, 由于灌木地上部分 调落物在灌从下的累积与分解, 以及由风力引起灌 从间表层土壤的侵蚀和土壤黏粉粒物质在灌从下的 沉积等过程，最终引起灌从下土壤养分的富集而形 成沃岛(Schlesinger et al., 1996; Whitford et al., 1997; 苏永中等, 2002; 陈广生等, 2003)。灌木植株密度、 冠幅大小、地形以及沙丘的固定程度等因素都会影 响灌木沃岛效应的强弱, 造成灌木下土壤养分的梯 度变化 (吴旭东等, 2016; 何佳等, 2018), 这为我们 研究灌木的内稳性特征提供了条件。因此, 本研究 通过调查科尔沈沙地不同类型沙地上优势固沙灌木 小叶锦鸡儿(Caragana microphylla)和盐高(Artemisia halodendron)叶片 $\mathrm{N} 、 \mathrm{P}$ 化学计量特征以及灌木下土 壤养分状况, 研究优势固沙灌木叶片 $\mathrm{N} 、 \mathrm{P}$ 化学计量 特征的变化及内稳性特征, 以期为科学认识科尔沁 沙地优势固沙灌木的生态适应性和固沙植被演变规 律提供依据。

\section{1 材料和方法}

\section{1 研究区概况}

研究区域位于内蒙古东北部科尔沈沙地中西部 的奈曼旗境内。地理位置为 $42.97^{\circ} \mathrm{N}, 120.73^{\circ} \mathrm{E}$, 海 拔高度约为 $360 \mathrm{~m}$ 。年平均气温 $6.5{ }^{\circ} \mathrm{C}$, 最热月(7月) 平均气温 $23.5{ }^{\circ} \mathrm{C}$, 最冷月 (1月)平均气温 $-13.2{ }^{\circ} \mathrm{C}$, 全年 $\geqslant 10{ }^{\circ} \mathrm{C}$ 的有效积温 $3200-3400{ }^{\circ} \mathrm{C}$, 无霜期 151 天, 极端最高气温 $39{ }^{\circ} \mathrm{C}$, 极端最低气温 $-29.3{ }^{\circ} \mathrm{C}$, 夏季无植被覆盖的沙丘表面最高温度可达57.2$60.0{ }^{\circ} \mathrm{C}$ 。年太阳辐射总量为 $5200-5400 \mathrm{MJ} \cdot \mathrm{m}^{-2}$, 年 降水量为360 mm左右, 主要集中在6-8月, 年蒸发 量 $1935 \mathrm{~mm}$, 属温带大陆型半干旱气候类型(李玉 霖等, 2005)。

该区的地貌类型以固定沙丘、半固定沙丘、流 动沙丘和丘间低地交错分布为主。优势植物以盐蒿、 小叶锦鸡儿等灌木以及狗尾草(Setaria viridis)、䊁隐 子草 (Cleistogenes squarrosa)、兴安胡枝子 (Lespedeza daurica) 和花苜宿 (Medicago ruthenica $=$ Melissitus ruthenicus)等一年生和多年生的杂类草为 
主(李玉霖等, 2005)。

\section{2 调查与取样}

在研究区内的流动沙丘、半固定沙丘、固定沙 丘和丘间低地等类型沙地上, 选取生长发育良好且 没有病虫害的盐蒿灌木79株和小叶锦鸡儿灌木77株, 7-8月每株灌木上采集成熟叶片20-30 g, 带回室内 在65 ${ }^{\circ} \mathrm{C}$ 下烘干 $48 \mathrm{~h}$, 烘干叶片研磨粉碎后保存。同 时在灌木冠幅内用土钻随机采集 5处0-15 $\mathrm{cm}$ 深度的 土壤样品, 混合均匀并带回室内自然阴干后过 $2 \mathrm{~mm}$ 土壤筛后待测。

\section{3 样品测试}

植物叶片 $\mathrm{N}$ 含量和土壤全 $\mathrm{N}$ 含量采用全自动凯 氏定氮仪(Kjeltec 8400, FOSS, Höganäs, Sweden)测 定, 叶片 $\mathrm{P}$ 含量和土壤全 $\mathrm{P}$ 含量采用钿锑抗比色法进 行测定。土壤速效 $\mathrm{N}$ 含量采用碱解扩散吸收法测定, 土壤速效 $\mathrm{P}$ 采用 $0.5 \mathrm{~mol} \cdot \mathrm{L}^{-1} \mathrm{NaHCO}_{3}$ 溶液提取, 然后 采用钼锑抗比色法测定含量(中国科学院南京土壤 研究所, 1978)。

\section{4 数据分析方法}

灌木叶片 N $\mathrm{P}$ 化学计量的内稳性利用Sterner和 Elser (2002)提出生物生态化学计量内稳性模型计 算。该模型指数公式为:

$$
Y=c X^{1 / H}
$$

其中, $Y$ 是灌木叶片 $\mathrm{N} 、 \mathrm{P}$ 含量及 $\mathrm{N}: \mathrm{P}, X$ 是土壤的 速效 $\mathrm{N}$ 、速效 $\mathrm{P}$ 含量及对应的 $\mathrm{N}: \mathrm{P}, c$ 为常数, $H$ 为植物 内稳性指数。

数据的正态分布采用One-Sample KolmogorovSmirnov Text进行检验，检验后不满足正态分布的 数据采用对数转化使其服从正态分布。通过ANOVA 分析盐高和小叶锦鸡儿间的叶片 $\mathrm{N}$ 含量、 $\mathrm{P}$ 含量和 $\mathrm{N}: \mathrm{P}$, 以及冠幅内土壤 $\mathrm{N}$ 含量、 $\mathrm{P}$ 含量、 $\mathrm{N}: \mathrm{P}$ 及速效 $\mathrm{N}$
含量、速效 $\mathrm{P}$ 含量、速效 $\mathrm{N}: \mathrm{P}$ 的差异。用回归分析拟 合盐蒿和小叶锦鸡儿叶片 $\mathrm{N}$ 含量、 $\mathrm{P}$ 含量、 $\mathrm{N}: \mathrm{P}$ 与土 壤速效 $\mathrm{N}$ 含量、速效 $\mathrm{P}$ 含量、速效 $\mathrm{N}: \mathrm{P}$ 之间的关系。 采用SPSS统计分析软件包(SPSS 16.0 for Windows, Chicago, USA)进行数据的统计分析。

\section{2 结果分析}

\section{1 盐蒿和小叶锦鸡儿叶片 $\mathrm{N} 、 \mathrm{P}$ 化学计量特征}

盐蒿叶片 $\mathrm{N} 、 \mathrm{P}$ 含量的变化范围为15.00-31.40和 2.86-4.53 g. kg ${ }^{-1}$, 算数平均值 (土标准误差) 分别为 $(23.24 \pm 0.49)$ 和 $(3.80 \pm 0.05) \mathrm{g} \cdot \mathrm{kg}^{-1}$ (表1)。相比较而 言, 小叶锦鸡儿叶片具有较高的 $\mathrm{N}$ 含量和较低的 $\mathrm{P}$ 含量 $(p<0.001$, 图1), N、P含量分别为 $(30.44 \pm 0.46)$ 和 $(1.96 \pm 0.04) \mathrm{g} \cdot \mathrm{kg}^{-1}$ 。盐蒿叶片的 $\mathrm{N}: \mathrm{P}$ 远低于小叶 锦鸡儿 $(p<0.001$, 图 1$)$, 算数平均值 ( \pm 标准误差)分 别为 $6.10 \pm 0.10$ 和 $15.86 \pm 0.29$ (表1)。

\section{2 盐蒿和小叶锦鸡儿冠幅内土壤 $N 、 P$ 化学计量 特征}

79株盐蒿冠幅内 $0-15 \mathrm{~cm}$ 深度土壤全 $\mathrm{N}$ 和全 $\mathrm{P}$ 含 量的变化范围分别为 $0.01-0.38$ 和 $0.00-0.11 \mathrm{~g} \cdot \mathrm{kg}^{-1}$, 平均值分别为 $(0.15 \pm 0.01)$ 和 $(0.05 \pm 0.00) \mathrm{g} \cdot \mathrm{kg}^{-1}$; 速 效 $\mathrm{N}$ 和速效 $\mathrm{P}$ 含量的变化范围分别为1.17-38.00和 $1.28-20.06 \mathrm{mg} \cdot \mathrm{kg}^{-1}$, 平均值分别为 $(15.77 \pm 1.00)$ 和 $(7.82 \pm 0.57) \mathrm{mg} \cdot \mathrm{kg}^{-1}$ (表2)。相比较而言, 小叶锦鸡 儿冠幅内 $0-15 \mathrm{~cm}$ 深度土壤具有较高的全 $\mathrm{N}$ 、全 $\mathrm{P}$ 、 速效 $\mathrm{N}$ 和速效 $\mathrm{P}$ 含量 $(p<0.001$, 图 2$)$, 全 $\mathrm{N}$ 和全 $\mathrm{P}$ 含 量分别为 $(0.23 \pm 0.02)$ 和 $(0.08 \pm 0.01) \mathrm{g} \cdot \mathrm{kg}^{-1}$; 速效 $\mathrm{N}$ 和速效 $\mathrm{P}$ 含量分别为 $(19.38 \pm 1.08)$ 和 $(9.74 \pm 0.55)$ $\mathrm{mg} \cdot \mathrm{kg}^{-1}$ 。但是盐蒿和小叶锦鸡儿的土壤 $\mathrm{N}: \mathrm{P}$ 和速效 $\mathrm{N}: \mathrm{P}$ 基本接近, 分别为 $3.10 \pm 0.26 、 3.08 \pm 0.21$ 和 $2.27 \pm 0.12 、 2.11 \pm 0.07$ (表2)。

表1 科尔沁沙地优势固沙灌木盐蒿和小叶锦鸡儿叶片 $\mathrm{N} 、 \mathrm{P}$ 含量和比值(平均值土标准误差)

Table 1 Nitrogen $(\mathrm{N})$ and phosphorus $(\mathrm{P})$ concentrations and their ratios in leaves of Artemisia halodendron and Caragana microphylla in Horqin Sandy Land $($ mean $\pm S E)$

\begin{tabular}{|c|c|c|c|c|c|c|c|}
\hline $\begin{array}{l}\text { 物种 } \\
\text { Species }\end{array}$ & $\begin{array}{l}\text { 指标 } \\
\text { Index }\end{array}$ & $\begin{array}{c}\text { 算数平均值 } \\
\text { Arithmetic average }\end{array}$ & $\begin{array}{l}\text { 中位数 } \\
\text { Median }\end{array}$ & $\begin{array}{l}\text { 众数 } \\
\text { Mode }\end{array}$ & $\begin{array}{c}\text { 变异系数 } \\
\text { Coefficient of } \\
\text { variation(\%) }\end{array}$ & $\begin{array}{c}\text { 极小值 } \\
\text { Minimum value }\end{array}$ & $\begin{array}{c}\text { 极大值 } \\
\text { Maximum value }\end{array}$ \\
\hline \multirow{3}{*}{$\begin{array}{l}\text { 盐蒿 } \\
\text { A. halodendron }\end{array}$} & $\mathrm{N}\left(\mathrm{g} \cdot \mathrm{kg}^{-1}\right)$ & $23.24 \pm 0.49$ & 24.50 & 27.00 & 18.71 & 15.00 & 31.40 \\
\hline & $\mathrm{P}\left(\mathrm{g} \cdot \mathrm{kg}^{-1}\right)$ & $3.80 \pm 0.05$ & 3.82 & 3.67 & 11.05 & 2.86 & 4.53 \\
\hline & $\mathrm{N}: \mathrm{P}$ & $6.10 \pm 0.10$ & 6.17 & 5.59 & 14.43 & 4.02 & 7.82 \\
\hline \multirow{3}{*}{$\begin{array}{l}\text { 小叶锦鸡儿 } \\
\text { C. microphylla }\end{array}$} & $\mathrm{N}\left(\mathrm{g} \cdot \mathrm{kg}^{-1}\right)$ & $30.44 \pm 0.46$ & 31.00 & 30.40 & 13.24 & 18.20 & 37.90 \\
\hline & $\mathrm{P}\left(\mathrm{g} \cdot \mathrm{kg}^{-1}\right)$ & $1.96 \pm 0.04$ & 1.97 & 1.97 & 17.86 & 1.24 & 2.69 \\
\hline & $\mathrm{N}: \mathrm{P}$ & $15.86 \pm 0.29$ & 15.71 & 11.28 & 15.82 & 11.28 & 24.33 \\
\hline
\end{tabular}



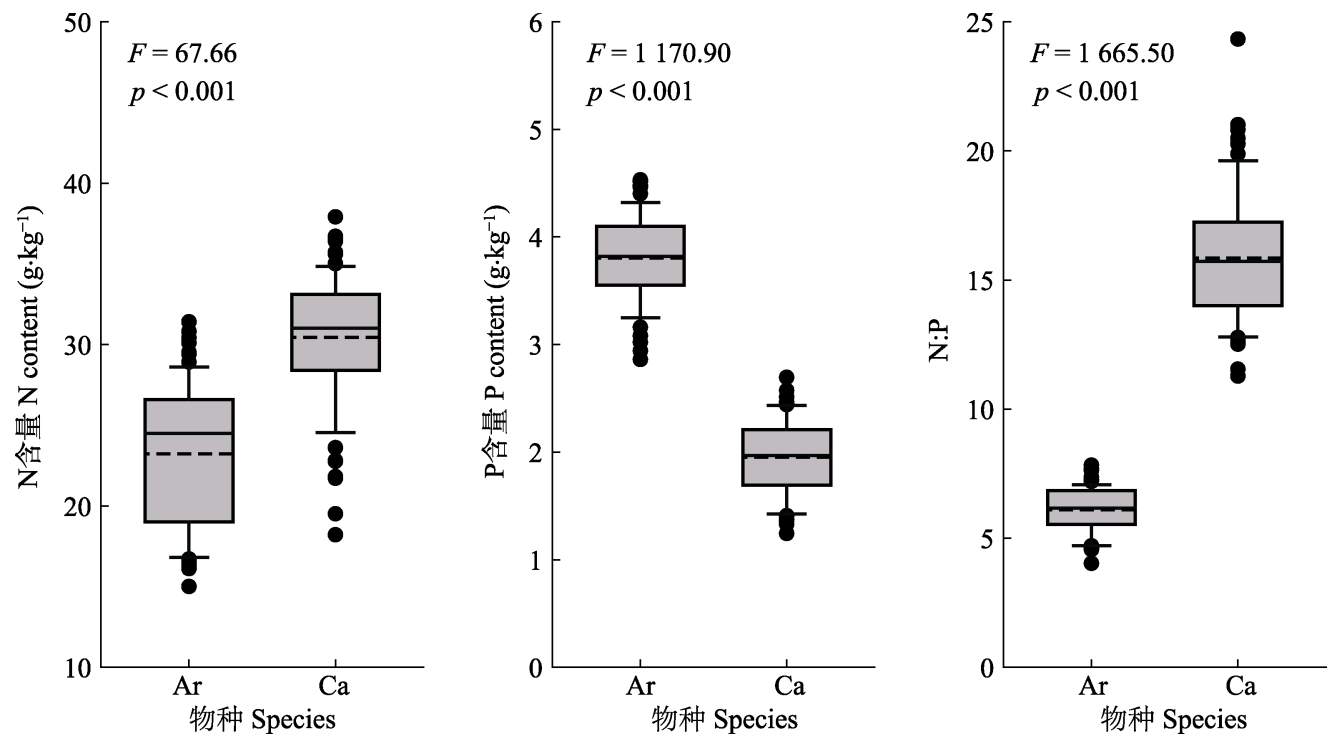

图1 科尔沁沙地优势固沙灌木盐蒿和小叶锦鸡儿叶片 $\mathrm{N} 、 \mathrm{P}$ 化学计量特征比较。 $p<0.001$ 表示盐蒿和小叶锦鸡儿的该指标在 0.001水平上差异显著; 误差线表示标准误差; Ar, Artemisia halodendron; Ca, Caragana microphylla。

Fig. 1 Comparisons of leaf nitrogen (N) and phosphorus (P) stoichiometry between Artemisia halodendron and Caragana microphylla in Horqin Sandy Land. Differences between Artemisia halodendron and Caragana microphylla are significant at $p<0.001$. Vertical bars represent standard errors. Ar, Artemisia halodendron; Ca, Caragana microphylla.

表2 科尔沁沙地优势固沙灌木盐蒿和小叶锦鸡儿冠幅内土壤 $\mathrm{N} 、 \mathrm{P}$ 化学计量特征(平均值土标准误差)

Table 2 Nitrogen $(\mathrm{N})$ and phosphorus (P) stoichiometry in soils beneath Artemisia halodendron and Caragana microphylla in Horqin Sandy Land (mean \pm SE)

\begin{tabular}{|c|c|c|c|c|c|c|c|}
\hline $\begin{array}{l}\text { 物种 } \\
\text { Species }\end{array}$ & $\begin{array}{l}\text { 指标 } \\
\text { Index }\end{array}$ & $\begin{array}{c}\text { 算数平均值 } \\
\text { Arithmetic average }\end{array}$ & $\begin{array}{l}\text { 中位数 } \\
\text { Median }\end{array}$ & $\begin{array}{l}\text { 众数 } \\
\text { Mode }\end{array}$ & $\begin{array}{c}\text { 变异系数 } \\
\text { Coefficient of } \\
\text { variation( } \%)\end{array}$ & $\begin{array}{c}\text { 极小值 } \\
\text { Minimum value }\end{array}$ & $\begin{array}{c}\text { 极大值 } \\
\text { Maximum value }\end{array}$ \\
\hline \multirow{6}{*}{$\begin{array}{l}\text { 盐蒿 } \\
\text { A. halodendron }\end{array}$} & $\mathrm{N}\left(\mathrm{g} \cdot \mathrm{kg}^{-1}\right)$ & $0.15 \pm 0.01$ & 0.14 & $0.17^{*}$ & 57.14 & 0.01 & 0.38 \\
\hline & $\mathrm{P}\left(\mathrm{g} \cdot \mathrm{kg}^{-1}\right)$ & $0.05 \pm 0.00$ & 0.05 & $0.03^{*}$ & 60.00 & 0.00 & 0.11 \\
\hline & $\mathrm{N}: \mathrm{P}$ & $3.10 \pm 0.26$ & 2.38 & 0.43 & 73.22 & 0.43 & 12.10 \\
\hline & 速效 N Available N $\left(\mathrm{mg} \cdot \mathrm{kg}^{-1}\right)$ & $15.77 \pm 1.00$ & 16.20 & 21.60 & 56.04 & 1.17 & 38.00 \\
\hline & 速效 P Available P (mg·kg $\left.{ }^{-1}\right)$ & $7.82 \pm 0.57$ & 6.80 & 9.80 & 64.32 & 1.28 & 20.06 \\
\hline & 速效 N:P Available N:P & $2.27 \pm 0.12$ & 2.20 & 3.60 & 46.70 & 0.59 & 5.77 \\
\hline \multirow{6}{*}{$\begin{array}{l}\text { 小叶锦鸡儿 } \\
\text { C. microphylla }\end{array}$} & $\mathrm{N}\left(\mathrm{g} \cdot \mathrm{kg}^{-1}\right)$ & $0.23 \pm 0.02$ & 0.21 & 0.02 & 69.56 & 0.02 & 0.65 \\
\hline & $\mathrm{P}\left(\mathrm{g} \cdot \mathrm{kg}^{-1}\right)$ & $0.08 \pm 0.01$ & 0.07 & 0.03 & 62.50 & 0.01 & 0.19 \\
\hline & $\mathrm{N}: \mathrm{P}$ & $3.08 \pm 0.21$ & 2.87 & 0.76 & 61.04 & 0.76 & 11.99 \\
\hline & 速效 N Available $\mathrm{N}\left(\mathrm{mg} \cdot \mathrm{kg}^{-1}\right)$ & $19.38 \pm 1.08$ & 16.88 & $9.43^{*}$ & 48.81 & 7.39 & 44.86 \\
\hline & 速效 P Available P (mg·kg $\left.{ }^{-1}\right)$ & $9.74 \pm 0.55$ & 8.60 & $6.10^{*}$ & 49.90 & 2.93 & 24.80 \\
\hline & 速效 N:P Available N:P & $2.11 \pm 0.07$ & 2.11 & $0.35^{*}$ & 27.49 & 0.35 & 3.91 \\
\hline
\end{tabular}

*表示有多个众数。

* indicating more than one mode.

\section{3 盐蒿和小叶锦鸡儿叶片的 $N 、 P$ 化学计量内} 稳性

用内稳性模型 $Y=c X^{1 / H}$ 模拟发现，盐蒿和小叶 锦鸡儿叶片 $\mathrm{N}$ 含量、 $\mathrm{P}$ 含量及 $\mathrm{N}: \mathrm{P}$ 均表现出显著的内 稳性特征 $(p<0.001$, 图3)。盐蒿叶片 $\mathrm{N}$ 含量、 $\mathrm{P}$ 含量 及 $\mathrm{N}: \mathrm{P}$ 的内稳性指数 $H$ 的大小表现为: $H_{\mathrm{P}}>H_{\mathrm{N}: \mathrm{P}}>H_{\mathrm{N}}$, 分别为 $8.19 、 7.90$ 和 4.20 。小叶锦鸡儿叶片 $\mathrm{N}$ 含量、 $\mathrm{P}$ 含量及 $\mathrm{N}: \mathrm{P}$ 的内稳性指数 $H$ 的大小表现为: $H_{\mathrm{N}: \mathrm{P}}>$ $H_{\mathrm{N}}>H_{\mathrm{P}}$, 分别为 $7.81 、 5.49$ 和 3.50 。同时, 小叶锦鸡
儿叶片 $\mathrm{N}$ 的内稳性高于盐蒿, 其叶片 $\mathrm{P}$ 的内稳性低 于盐蒿, 但它们 $\mathrm{N}: \mathrm{P}$ 的内稳性基本相近。

\section{3 讨论}

\section{1 优势固沙灌木叶片及冠幅内土壤 $N$ 、P含量和 比值}

$\mathrm{N} 、 \mathrm{P}$ 作为植物生长发育所必需的营养元素，其 化学计量特征能反映植物的养分利用状况和元素平 衡特点。本研究发现，与盐蒿相比，作为豆科植物的 

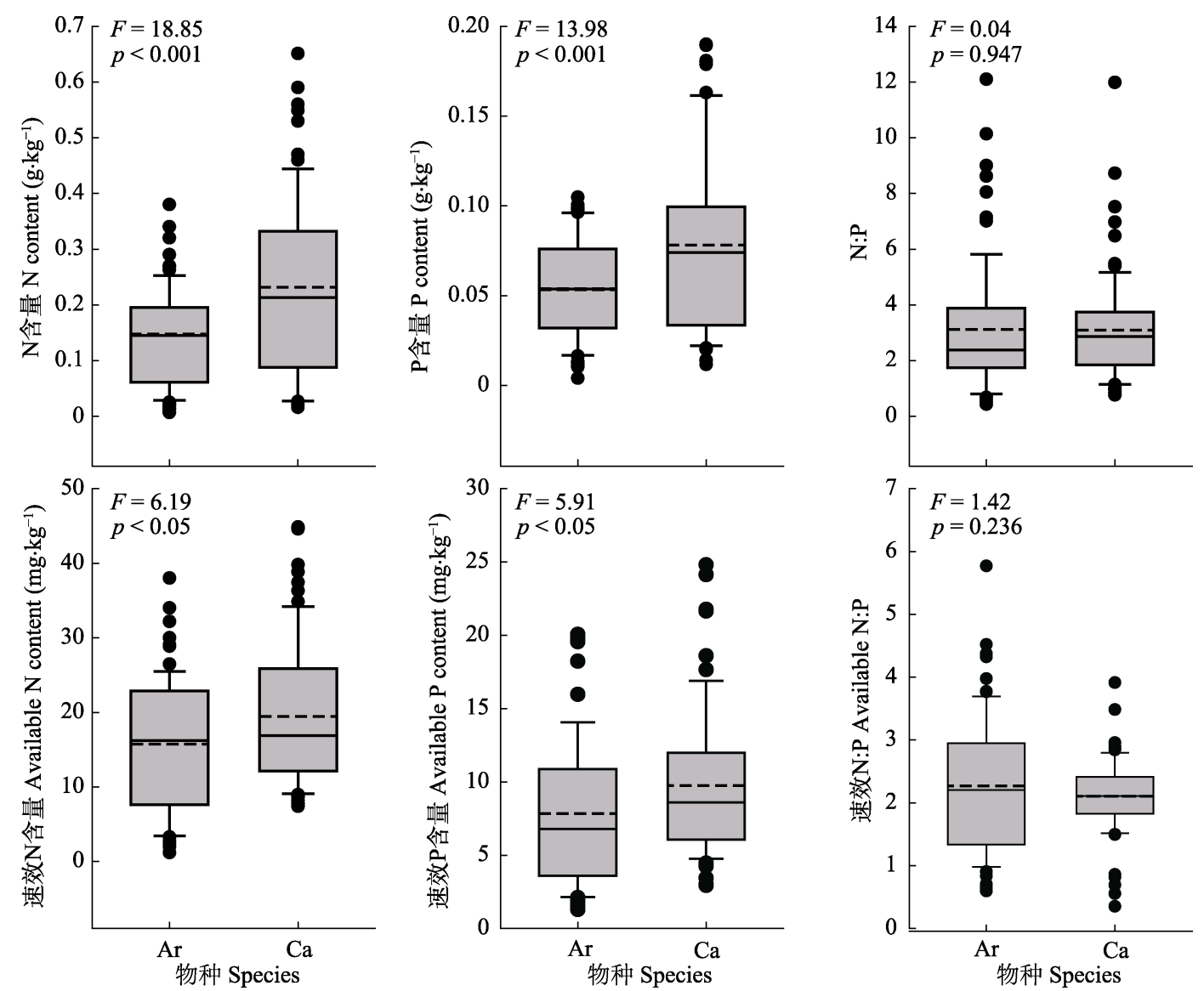

图2 科尔沈沙地优势固沙灌木盐蒿和小叶锦鸡儿土壤 $\mathrm{N} 、 \mathrm{P}$ 化学计量特征比较。 $p<0.001$ 表示盐高和小叶锦鸡儿的该指标在 0.001水平上差异显著; 误差线表示标准误差; Ar, Artemisia halodendron; Ca, Caragana microphylla。

Fig. 2 Comparisons of soil nitrogen $(\mathrm{N})$ and phosphorus (P) stoichiometry between Artemisia halodendron and Caragana microphylla in Horqin Sandy Land. Differences between Artemisia halodendron and Caragana microphylla are significant at $p<0.001$. Vertical bars represent standard errors. Ar, Artemisia halodendron; Ca, Caragana microphylla.

小叶锦鸡儿具有较高的叶片 $\mathrm{N}$ 含量, 而其 $\mathrm{P}$ 含量约 为盐蒿叶片 $\mathrm{P}$ 含量的 $1 / 2$, 这与已有的研究结果相一 致(朱军涛等, 2010; 宁志英等, 2017)。本研究中, 盐 蒿和小叶锦鸡儿叶片 $\mathrm{N}: \mathrm{P}$ 分别为 6.10 和 $15.86, \mathrm{Ko}-$ erselman和Meuleman (1996) 认为, 当植物叶片 $\mathrm{N}: \mathrm{P}$ 小于14时, 植物生长主要受 N限制; 当N:P大于16时, 植物生长主要受P限制; N:P在14-16范围内时, 植物 受N和P共同限制。依据该理论，本研究中盐蒿受N 的限制，而小叶锦鸡儿受N、P共同限制，但受N限制 的作用较小，这主要依赖于其自身的共生固氮作 用。因此，小叶锦鸡儿在受 $\mathrm{N}$ 限制的荒漠环境中比盐 蒿更具优势，对沙地生态恢复具有重要的意义。

土壤养分供应状况直接影响着植物光合作用及 矿物质代谢等生态过程, 其养分组成对植物生长具 有重要作用(宾振钧等, 2014)。本研究中, 由于该地 区沙漠化的发展导致土壤养分大量流失, 盐蒿和小 叶锦鸡儿灌从内土壤全 $\mathrm{N} 、$ 全P含量均低于中国表层
土壤的研究结果(全N: 1.86; 全P: 0.78)(Tian et al., 2010)。然而, 与本研究区其他研究结果相比, 灌从 内土壤具有较高的全 $\mathrm{N} 、$ 全 $\mathrm{P} 、$ 速效 $\mathrm{N}$ 及速效 $\mathrm{P}$ 含量(熊 炳桥等, 2017)。灌丛土壤养分得益于灌木叶片对大 气降尘、土壤细颗粒物质及其他有机物碎屑的拦截 产生的“沃岛效应” (Wezel et al., 2000), 以及灌木通 过叶片调落物及根系周转向土壤中补充的有机质 (Fisher, 1995)。苏永中等(2004)也发现在流动沙丘上 人工定植小叶锦鸡儿后, 其土壤理化性状明显改 善。本研究发现, 小叶锦鸡儿灌从土壤全 $\mathrm{N}$ 、全 $\mathrm{P} 、$ 速效 $\mathrm{N}$ 及速效 $\mathrm{P}$ 含量均显著高于盐蒿 $(p<0.001)$, 这 是小叶锦鸡儿的共生固氮作用与其叶片调落物对土 壤的归还过程相互作用的结果。然而, 灌从土壤 $\mathrm{N}: \mathrm{P}$ 及速效 $\mathrm{N}: \mathrm{P}$ 在二者之间无显著差异。土壤P主要受土 壤母质的影响，而土壤 $\mathrm{N}$ 除受土壤母质的影响外， 还受调落物分解及植物根系输入的影响(Reich \& Oleksyn, 2004)。本研究中, 小叶锦鸡儿分布在P含量 

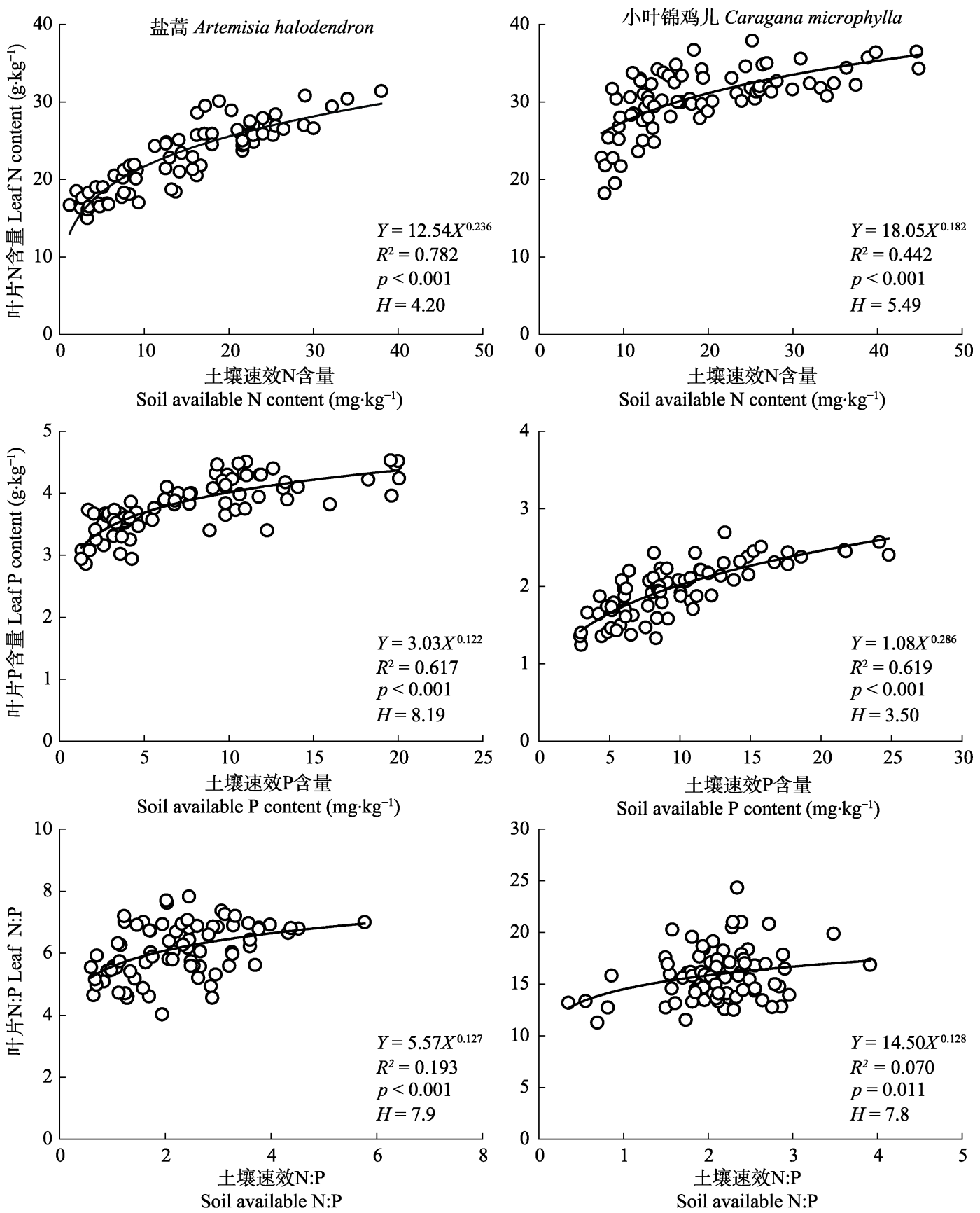

图3 科尔沁沙地优势固沙灌木叶片 $\mathrm{N} 、 \mathrm{P}$ 及比值与对应土壤速效 $\mathrm{N} 、 \mathrm{P}$ 及其比值的关系。 $H$, 内稳性指数。

Fig. 3 Relationships of leaf N, P, and N:P with soil N, P, and N:P in two dominant shrubs in Horqin Sandy Land. H, stoichiometric homoeostasis indexes.

较高的土壤中, 因其叶片 $\mathrm{N}$ 含量较高, 其向土壤输 入的调落物亦具有较高的 $\mathrm{N}$ 含量; 而盐蒿分布在 $\mathrm{P}$ 含量较低的土壤中, 其具有较低的叶片 $\mathrm{N}$ 含量, 其 向土壤输入的调落物 $\mathrm{N}$ 含量较低, 因此导致土壤 $\mathrm{N}: \mathrm{P}$ 及速效 $\mathrm{N}: \mathrm{P}$ 在这两种灌木之间差异不大。

\section{2 优势固沙灌木的内稳性特征}

内稳性是生物对环境变化所形成的生理生化适 应机制的体现(Elser et al., 2010)。植物的内稳性特征
是生态化学计量学的核心概念, 内稳性的高低体现 了植物对土壤养分供应变化的内在适应机制(Yu et al., 2010)。生态化学计量内稳性模型 $Y=c X^{1 / H}$ (Sterner \& Elser, 2002)为量化植物体内元素含量及 比值对环境变化的响应程度提供了新途径。Persson 和Kato (2010) 认为植物内稳性特征可用内稳性指数 $H$ 进行界定，按内稳性指数可将植物划分为 4 种类型: $H>4$ 为稳态型; $2<H<4$ 为弱稳态型; $4 / 3<H<2$ 为 
弱敏感型; $H<4 / 3$ 为敏感型。在对维管植物内稳性 的研究中, 一些学者通过实验测算 $H$ 来表征植物的 内稳性大小。例如, Li等(2016)对不同沙化程度羊草 草地的研究发现, 羊草的内稳性指数在1.53-15.92 之间, 属强(2009)对内蒙古草原12个物种的研究发 现, N、P及 $\mathrm{N}: \mathrm{P}$ 的内稳性指数具有较大的变异范围, 在1.93-14.49之间。本研究中盐蒿和小叶锦鸡儿叶片 $\mathrm{N} 、 \mathrm{P}$ 及 $\mathrm{N}: \mathrm{P}$ 内稳性指数分别在 $4.20-8.19$ 和3.50-7.80 之间。

早在生态化学计量内稳性模型提出之前, Garnier (1998)指出植物元素与土壤中对应养分含量间 呈显著相关关系时, 该养分即为限制性养分。该理 论亦是基于特定环境中植物与土壤间的相互作用而 提出的, 是植物养分吸收的内在属性及土壤养分供 应状况的综合体现(樊晓勇, 2012)。因此, 植物内稳 性的高低与养分限制间具有密切关联性。本研究中, 盐蒿和小叶锦鸡儿间的叶片的内稳性具有一定的差 异。就N含量而言, 小叶锦鸡儿叶片的内稳性高于盐 蒿, 主要是由于小叶锦鸡儿自身的共生固氮作用使 其在 $\mathrm{N}$ 限制的环境中仍能保持较为稳定的 $\mathrm{N}$ 含量, 而盐蒿叶片 $\mathrm{N}$ 含量更易随土壤 $\mathrm{N}$ 含量的变化而发生 变化, 这与Guo等(2017)的研究结果相一致。就P含 量而言, 盐蒿的内稳性指数高于小叶锦鸡儿, 表明 小叶锦鸡儿叶片 $\mathrm{P}$ 含量更易受土壤 $\mathrm{P}$ 含量的影响, 其 生长受P限制。尽管小叶锦鸡儿受 $\mathrm{P}$ 的影响较大, 而 盐蒿受 $\mathrm{N}$ 的影响较大, 它们的 $\mathrm{N}: \mathrm{P}$ 内稳性指数基本 相等, 且 $\mathrm{N}: \mathrm{P}$ 受土壤养分含量变化的影响不大。

同时, 盐蒿和小叶锦鸡儿叶片 $\mathrm{N}$ 含量、 $\mathrm{P}$ 含量及 $\mathrm{N}: \mathrm{P}$ 的内稳性特征具有一定的差异性。盐高叶片的 内稳性表现为 $H_{\mathrm{P}}>H_{\mathrm{N}: \mathrm{P}}>H_{\mathrm{N}}$, 意味着其叶片 $\mathrm{P}$ 含量 能保持较强的内稳性而受土壤P含量影响较小, 而 $\mathrm{N}$ 含量随土壤 $\mathrm{N}$ 含量的变化而变化, 导致其 $\mathrm{N}: \mathrm{P}$ 亦随 之发生变化, 这主要是由于研究区内沙化草地受 $\mathrm{N}$ 的限制作用较大。小叶锦鸡儿叶片的内稳性表现为 $H_{\mathrm{N}: \mathrm{P}}>H_{\mathrm{N}}>H_{\mathrm{P}}$ 。小叶锦鸡儿自身的固氮特性使其在 $\mathrm{N}$ 素缺乏的沙化草地中获得充足的 $\mathrm{N}$, 其叶片 $\mathrm{N}$ 含量 受土壤养分含量变化的影响较小, 相对比较稳定。 尽管小叶锦鸡儿叶片 $\mathrm{P}$ 含量受土壤养分含量的影响 较大, 其 $\mathrm{N}: \mathrm{P}$ 基本不受土壤 $\mathrm{P}$ 含量的影响, 表现出极 强的内稳性, 其原因可能是: 1)小叶锦鸡儿自身具 有较强的养分调控能力, 这可能是其在长期进化过 程中对贫痊环境适应的结果; 2)中国土壤P含量变异
幅度较大，从湿润区向干旱半干旱区呈增加趋势 (汪涛等, 2008), 说明在中国区域内干旱半干旱区土 壤具有相对较高的土壤P含量, 因此该地区植物生 长受 $\mathrm{P}$ 限制的作用较小。植物组织中 $\mathrm{N}$ 浓度的增加一 般都伴随着 $\mathrm{P}$ 浓度的增加, 较高的 $\mathrm{N}: \mathrm{P}$ 内稳性意味着 小叶锦鸡儿叶片 $\mathrm{N}: \mathrm{P}$ 的变化比 $\mathrm{N}$ 和 $\mathrm{P}$ 各自的变化小, 在其生长过程中对 $\mathrm{N} 、 \mathrm{P}$ 吸收量的变化较为一致, 表 明小叶锦鸡儿具有保守的养分利用策略(Sterner \& Elser, 2002)。夷强(2009)指出高的内稳性和保守的 养分利用策略是植物在干旱贫痊的关键中得以生存 的关键。因此, 相比于盐蒿, 小叶锦鸡儿内稳性的调 节能力使其在多变的环境中保持较好的生长状态, 具有更强的适应性及竞争力。

本研究中植物叶片内稳性的高低与其受养分限 制类型及限制程度具有一致的变化趋势。土壤养分 供应状况会影响植物内稳性的高低。因此, 植物化 学计量内稳性的高低除了受物种、器官、元素种类 及生长发育阶段等因素的影响外(属强, 2009), 还与 土壤限制性养分的种类及受限制程度有关。尽管判 断植物限制性养分的Koerselman理论(Koerselman \& Meuleman, 1996)目前在水生及陆地生态系统中 均得到广泛应用, 但其因生态系统类型、研究区域 及物种种类的差异性而具有一定的局限性, 用统一 标准来衡量生态系统的元素限制是不合适的。因此 通过植物化学计量内稳性的高低来判断植物养分限 制或许更为可靠。

必须指出的是, 本研究中, 灌木自身所形成的 “沃岛效应”, 加上在调查过程中选择了不同固定程 度沙丘上的灌木植株, 使所研究土壤形成了养分梯 度。然而, 由于研究区沙漠化的发展导致土壤养分 的大量流失，不能保证养分梯度上最大养分浓度时 植物叶片中养分含量已达到稳定状态。因此, 本研 究中所调查的养分梯度仅反映了沙质草地的实际状 况, 在分析这两种灌木植物内稳性过程中可能存在 一定的缺陷。若要深入解析盐蒿和小叶锦鸡儿内稳 性机理, 还应进一步进行养分添加实验来验证。

\section{4 结论}

本研究发现, 优势固沙灌木盐蒿和小叶锦鸡儿 的化学计量内稳性特征明显不同。盐蒿叶片 N、P化 学计量内稳性指数表现为 $H_{\mathrm{P}}>H_{\mathrm{N}: \mathrm{P}}>H_{\mathrm{N}}$, 说明盐蒿 更易受土壤 $\mathrm{N}$ 的限制; 小叶锦鸡儿叶片 $\mathrm{N} 、 \mathrm{P}$ 化学计 
量内稳性指数表现为 $H_{\mathrm{N}: \mathrm{P}}>H_{\mathrm{N}}>H_{\mathrm{P}}$, 意味着小叶锦 鸡儿更易受土壤的限制。共生固氮作用补充的 $\mathrm{N}$ 使 得小叶锦鸡儿具有较强的 $\mathrm{N}$ 内稳性。因此, 在 $\mathrm{N}$ 含量 较低的沙化草地, $H_{\mathrm{N}}$ 较高的固沙灌木小叶锦鸡儿更 能适应环境而成为优势种, 进而在沙地恢复的早期 阶段为裸露沙地提供一定的覆盖度, 同时可能改善 土壤的理化性质。然而, 由于小叶锦鸡儿额外吸收 的 $\mathrm{N}$, 使其生长过程可能易受 $\mathrm{P}$ 的限制, 因此在沙地 恢复过程中应注意土壤P的供应。

\section{参考文献}

Aerts R, Chapin FS (2000). The mineral nutrition of wild plants revisited: A re-evaluation of processes and patterns. Advances in Ecological Research, 30, 1-67.

Andersen T, Elser JJ, Hessen DO (2004). Stoichiometry and population dynamics. Ecology Letters, 7, 884-900.

Bin ZJ, Wang JJ, Zhang WP, Xu DH, Cheng XH, Li KJ, Cao DH (2014). Effects of $\mathrm{N}$ addition on ecological stoichiometric characteristics in six dominant plant speciesof alpine meadow on the Qinghai-Xizang Plateau, China. Chinese Journal of Plant Ecology, 38, 231-237. [宾振钧, 王 静静, 张文鹏, 徐当会, 程雪寒, 李柯杰, 曹德昊 (2014). 氮肥添加对青藏高原高寒草甸6个群落优势种 生态化学计量学特征的影响. 植物生态学报, 38 , 231-237.]

Chen GS, Zeng DH, Chen FS, Fan ZP, Geng HD (2003). A research review on "fertile islands" of soils under shrub canopy in arid and semi-arid regions. Chinese Journal of Applied Ecology, 14, 2295-2300. [陈广生, 曾德慧, 陈伏 生, 范志平, 耿海东 (2003). 干旱和半干旱地区灌木下 土壤“肥岛”研究进展. 应用生态学报, 14, 2295-2300.]

Chen ZQ, Chen ZB, Yan XY, Bai LY (2016). Stoichiometric mechanisms of Dicranopteris dichotoma, growth and resistance to nutrient limitation in the Zhuxi watershed in the red soil hilly region of China. Plant \& Soil, 398, 367-379.

Daufresne T, Loreau M (2001). Ecological stoichiometry, primary producer-decomposer interactions, and ecosystem persistence. Ecology, 82, 3069-3082.

Elser JJ, Bracken MES, Cleland EE, Gruner DS, Harpole WS, Hillebrand H, Ngai JT, Seabloom EW, Shurin JB, Smith JE (2007). Global analysis of nitrogen and phosphorus limitation of primary producers in freshwater, marine and terrestrial ecosystems. Ecology Letters, 10, 1135-1142.

Elser JJ, Fagan WF, Kerkhoff AJ, Swenson NG, Enquist BJ (2010). Biological stoichiometry of plant production: Metabolism, scaling and ecological response to global change. New Phytologist, 186, 593-608.

Fan XY (2012). Spatial Variation in Nutrient of Dominate Plant and Ecological Stoichiometry from Laohu Gou of Qilian Mountin. Master degree dissertation, Lanzhou Univirsity, Lanzhou. [樊晓勇 (2012). 祁连山老虎沟优
势植物的养分空间变化与生态化学计量学研究. 硕士 学位论文, 兰州大学, 兰州.]

Fisher RF (1995). Amelioration of degraded rain forest soils by plantations of native trees. Soil Science Society of America Journal, 59, 544-549.

Frost PC, Elser JJ (2002). Growth responses of littoral mayflies to the phosphorus content of their food. Ecology Letters, 5, 232-240.

Garnier E (1998). Interspecific Variation in Plasticity of Grasses in Response to Nitrogen Supply. Cambridge University Press, Cambridge, UK. 155-181.

Guo YP, Yang X, Schob C, Jiang YX, Tang ZY (2017). Legume shrubs are more nitrogen-homeostatic than non-legume shrubs. Frontiers in Plant Science, 8, 1662.

Han WX, Fang JY, Guo DL, Zhang Y (2005). Leaf nitrogen and phosphorus stoichiometry across 753 terrestrial plant species in China. New Phytologist, 168, 377-385.

He J, Hu YF, Shu XY, Wang Q, Jia AD, Yan X (2018). Effect of Salix cupularis plantations on soil stoichiometry and stocks in the alpine-cold desert of northwestern Sichuan. Acta Prataculturae Sinica, 27, 27-33. [何佳, 胡玉福, 舒 向阳, 王琴, 贾安都, 严星 (2018). 川西北高寒沙地不 同年限高山柳土壤生态化学计量及储量变化特征. 草 业学报, 27, 27-33.]

Hessen DO, Agren GI, Anderson TR, Elser JJ, De Ruiter PC (2004). Carbon, sequestration in ecosystems: The role of stoichiometry. Ecology, 85, 1179-1192.

Hobbie SE, Vitousek PM (2000). Nutrient limitation of decomposition in hawaiian forests. Ecology, 81, 1867-1877.

Institute of Soil Science, Chinese Academy of Sciences (1978). Physical and Chemical AnalysisMethods of Soil. Shanghai Science and Technology Press, Shanghai. [中国科学院南 京土壤研究所 (1978). 土壤理化分析. 上海科学技术出 版社, 上海.]

Jeyasingh PD, Weider LJ, Sterner RW (2009). Geneticallybased trade-offs in response to stoichiometric food quality influence competition in a keystone aquatic herbivore. Ecology Letters, 12, 1229-1237.

Jiang LL, Zeng CS, Shao JJ, Zhou XH (2017). Plant nutrient dynamics and stoichiometric homeostasis of invasive species Spartina alternifloraand native Cyperus malaccensis var. brevifolius in the Minjiang River estuarine wetlands. Chinese Journal of Plant Ecology, 41, 450-460. [蒋利玲, 曾从盛, 邵钧昫, 周旭辉 (2017). 闽江河口入侵种互花 米草和本地种短叶茫芏的养分动态及植物化学计量内 稳性特征. 植物生态学报, 41, 450-460.]

Koerselman W, Meuleman AF (1996). The vegetation N:P ratio: A new tool to detect the nature of nutrient limitation. Journal of Applied Ecology, 33, 1441-1450.

Koojiman SALM (1995). The stoichiometry of animal energetics. Journal of Theoretical Biology, 177, 139-149.

Li Y, Li Q, Guo D, Liang S, Wang Y (2016). Ecological stoichiometry homeostasis of Leymus chinensis, in degraded 
grassland in western Jilin Province, NE China. Ecological Engineering, 90, 387-391.

Li YL, Cui JH, Su YZ (2005). Specific leaf area and leaf dry matter content of some plants in different dune habitats. Acta Ecologica Sinica, 25, 304-311. [李玉霖, 崔建垣, 苏永中 (2005). 不同沙丘生境主要植物比叶面积和叶 干物质含量的比较. 生态学报, 25, 304-311.]

Ning ZY, Li YL, Yang HL, Sun DC, Bi JD (2017). Carbon, nitrogen and phosphorus stoichiometry in leaves and fine roots of dominant plants in Horqin Sandy Land. Chinese Journal of Plant Ecology, 41, 1069-1080. [宁志英, 李玉 霖, 杨红玲, 孙殿超, 毕京东 (2017). 科尔沁沙地主要 植物细根和叶片碳、氮、磷化学计量特征. 植物生态学 报, 41, 1069-1080.]

Persson J, Kato S (2010). To be or not to be what you eat: Regulation of stoichiometric homeostasis among autotrophs and heterotrophs. Oikos, 119, 741-751.

Qu WL, Yang XP, Zhang CT, Wei B (2015). Shrub-mediated "fertile island" effects in arid and semi-arid grassland. Acta Prataculturae Sinica, 24, 201-207. [瞿王龙, 杨小鹏, 张存涛, 魏冰 (2015). 干旱、半干旱地区天然草原灌木 及其肥岛效应研究进展. 草业学报, 24, 201-207.]

Reich PB, Oleksyn J (2004). Global patterns of plant leaf N and $\mathrm{P}$ in relation to temperature and latitude. Proceedings of the National Academy of Sciences of the United States of America, 101, 11001-11006.

Reiners WA (1986). Complementary models for ecosystems. The American Naturalist, 127, 59-73.

Schlesinger WH, Raikes JA, Hartley AE, Cross AF (1996). On the spatial pattern of soil nutrients in desert ecosystems. Ecology, 77, 364-374.

Sterner RW, Elser JJ (2002). Ecological Stoichiometry: The Biology of Elements from Molecules to the Biosphere. Princeton University Press, Princeton.

Su YZ, Zhao HL, Zhang TH (2002). Influencing mechanism of several shrubs and subshrubs on soil fertility in Keerqin sandy land. Chinese Journalof Applied Ecology, 13, 802-806. [苏永中, 赵哈林, 张铜会 (2002). 几种灌木、 半灌木对沙地土壤肥力影响机制的研究. 应用生态学 报, 13, 802-806.]

Su YZ, Zhao HL, Zhang TH, Li YL (2004). Characteristics of plant community and soil properties in the plantation chronosequence of Caragana microphylla in Horqin Sandy Land. Acta Phytoecologica Sinica, 28, 93-100. [苏 永中, 赵哈林, 张铜会, 李玉霖 (2004). 科尔沁沙地不 同年代小叶锦鸡儿人工林植物群落特征及其土壤特性. 植物生态学报, 28, 93-100.]

Sundareshwar PV, Morris JT, Koepfler EK, Fornwalt B (2003). Phosphorus limitation of coastal ecosystem processes. Science, 299, 563.

Tian H, Chen G, Zhang C, Melillo JM, Hall CAS (2010). Pattern and variation of $\mathrm{C}: \mathrm{N}: \mathrm{P}$ ratios in China's soils: A synthesis of observational data. Biogeochemistry, 98, 139-151.

Vitousek PM (1999). Nutrient limitation to nitrogen fixation in

www.plant-ecology.com young volcanic sites. Ecosystems, 2, 505-510.

Wang T, Yang YH, Ma WH (2008). Storage, patterns and environmental controls of soil phosphorus in China. Acta Scientiarum Naturalium Universitatis Pekinensis, 44, 945-952. [汪涛, 杨元合, 马文红 (2008). 中国土壤磷 库的大小、分布及其影响因素. 北京大学学报(自然科学 版), 44, 945-952.]

Westheimer FH (1987). Why nature chose phosphates. Science, $235,1173$.

Wezel A, Rajot JL, Herbrig C (2000). Influence of shrubs on soil characteristics and their function in Sahelian agro-ecosystems in semi-arid Niger. Journal of Arid Environments, 44, 383-398.

Whitford WG, Anderson J, Rice PM (1997). Stemflow contribution to the "fertile island" effect in creosotebush, Larrea tridentata. Journal of Arid Environments, 35, 451-457.

Wu XD, Song NP, Pan J (2016). Effect of shrub (Caragana intermedia) encroachment under different sandy habitats on contentand distribution of soil organic carbon in desert grassland. Transactions of the Chinese Society of Agricultural Engineering, 32, 115-121. [吴旭东, 宋乃平, 潘军 (2016). 不同沙地生境下柠条灌丛化对草地土壤有机碳 含量及分布的影响. 农业工程学报, 32, 115-121.]

Xiong BQ, Zhao LY, Zhang J, Li YQ, Chen HB, Li FR (2017). Relationship between the soil andstanding vegetation changes during grassland desertification process. Ecology and Environmental Sciences, 26, 400-407. [熊炳桥, 赵丽 娅, 张劲, 李艳蓄, 陈红兵, 李锋瑞 (2017). 草地沙漠 化过程中土壤与地上植被的变化及其相互关系. 生态 环境学报, 26, 400-407.]

Yan JH, Li K, Peng XJ, Huang ZL, Liu SZ, Zhang QM (2015). The mechanism for exclusion of Pinus massoniana during the succession in subtropical forest ecosystems: Light competition or stoichiometric homoeostasis? Scientific Reports, 5, 10994. DOI: 10.1038/srep10994.

Yu Q (2009). Ecological Stoichiometric Study on VascularPlants in the Inner Mongolia Steppe. PhD dissertation, Institute of Botany, Chinese Academy of Sciences, Beijing. [座强 (2009). 内蒙古草原植物化学计量生态学研究. 博士学位论文, 中国科学院植物研究所, 北京.]

Yu Q, Chen QS, Elser JJ, Cease A, He NP, Wu HH, Zhang GM, Wu JG, Bai YF, Han XG (2010). Linking stoichiometric homeostasis with ecosystem structure, functioning, and stability. Ecology Letters, 13, 1390.

Zhu JT, Li XY, Zhang XM, Lin LS, Yang SG (2010). Nitrogen allocation and partitioning within a leguminous and two non-leguminous plant species growing at the southern fringe of China's Taklamakan Desert. Chinese Journal of Plant Ecology, 34, 1025-1032. [朱军涛, 李向义, 张希 明, 林丽莎, 杨尚功 (2010). 塔克拉玛干沙漠南缘豆科 与非豆科植物的氮分配. 植物生态学报, 34, 1025-1032.] 